\title{
A clue towards improving the European Society of Medical Oncology risk group classification in apparent early stage endometrial cancer? Impact of lymphovascular space invasion
}

\author{
S Bendifallah*,1,2, G Canlorbe ${ }^{1}$, E Raimond ${ }^{3}, \mathrm{D} \mathrm{Hudry}^{4}, \mathrm{C} \mathrm{Coutant}^{4}$, O Graesslin ${ }^{3}, \mathrm{C}$ Touboul $^{5}, \mathrm{~F} \mathrm{Huguet}^{6}$, \\ A Cortez ${ }^{7}$, E Darai ${ }^{1,8}$ and M Ballester ${ }^{1,8}$ \\ ${ }^{1}$ Department of Obstetrics and Gynaecology, Tenon University Hospital, Assistance Publique des Hôpitaux de Paris (AP-HP), \\ University Pierre and Marie Curie, Paris 6, Institut Universitaire de Cancérologie (IUC), Paris, France; ${ }^{2}$ INSERM UMR S 707, \\ 'Epidemiology, Information Systems, Modeling', University Pierre and Marie Curie, Paris, France; ${ }^{3}$ Department of Obstetrics and \\ Gynaecology, Institute Alix de Champagne University Hospital, Reims, France; ${ }^{4}$ Centre de lutte contre le cancer Georges François \\ Leclerc, Dijon, France; ${ }^{5}$ Department of Obstetrics and Gynecology, Centre Hospitalier Intercommunal, Créteil, France; \\ ${ }^{6}$ Department of Radiation Oncology, Tenon University Hospital, University Pierre and Marie Curie, Paris, France; ${ }^{7}$ Department \\ of Pathology, Tenon University Hospital, University Pierre and Marie Curie, Paris, France and ${ }^{8}$ INSERM UMR S 938, University Pierre \\ et Marie Curie, Paris, France
}

Background: Lymphovascular space invasion (LVSI) is one of the most important predictors of nodal involvement and recurrence in early stage endometrial cancer (EC). Despite its demonstrated prognostic value, LVSI has not been incorporated into the European Society of Medical Oncology (ESMO) classification. The aim of this prospective multicentre database study is to investigate whether it may improve the accuracy of the ESMO classification in predicting the recurrence risk.

Methods: Data of 496 patients with apparent early-stage EC who received primary surgical treatment between January 2001 and December 2012 were abstracted from prospective multicentre database. A modified ESMO classification including six risk groups was created after inclusion of the LVSI status in the ESMO classification. The primary end point was the recurrence accuracy comparison between the ESMO and the modified ESMO classifications with respect to the area under the receiver operating characteristic curve (AUC).

Results: The recurrence rate in the whole population was 16.1\%. The median follow-up and recurrence time were 31 (range: 1-152) and 27 (range: 1-134) months, respectively. Considering the ESMO modified classification, the recurrence rates were $8.2 \%$ (8 out of 98), $23.1 \%$ ( 15 out of 65$), 25.9 \%$ (15 out of 58 ), and $45.1 \%$ (28 out of 62) for intermediate risk/LVSI - , intermediate risk/LVSI + , high risk/ LVSI - , and high risk/LVSI +, respectively $(P<0.001)$. In the low risk group, LVSI status was not discriminant as only $7.0 \%$ (14 out of 213) had LVSI + . The staging accuracy according to AUC criteria for ESMO and ESMO modified classifications were of $0.71(95 \% \mathrm{Cl}$ : $0.68-0.74)$ and 0.74 (95\% Cl: 0.71-0.77), respectively.

Conclusions: The current modified classification could be helpful to better define indications for nodal staging and adjuvant therapy, especially for patients with intermediate risk EC.

*Correspondence: Dr S Bendifallah; E-mail: sofiane.bendifallah@yahoo.fr

Received 4 February 2014; revised 30 March 2014; accepted 10 April 2014; published online 8 May 2014

(c) 2014 Cancer Research UK. All rights reserved 0007-0920/14 
Endometrial cancer (EC) is a major cause of morbidity and mortality for patients worldwide. Although its incidence differs throughout the world, it is the most common cancer of the female genital tract and the fourth most common cancer in North America and Europe (Jemal et al, 2010; Colombo et al, 2013). Early-stage EC restricted to the uterus represents nearly $80 \%$ of all cases (Creasman et al, 1987, 2006). The primary surgical treatment involves removal of uterus, tubes, and ovaries, with or without nodal pelvic or para-aortic staging (Creasman et al, 1987; Keys et al, 2004). The estimated 5-year overall survival (OS) for earlystage EC is $95 \%$ but decreases substantially to $67.0 \%$ and $15.9 \%$ for local and distant disease, respectively (Creutzberg et al, 2000; Randall et al, 2006; Benedetti Panici et al, 2008; ASTEC study group et al, 2009).

Recurrence has been reported to occur in 15-20\% of early-stage EC (Mariani et al, 2002; Nout et al, 2010). Several epidemiological and histological prognostic markers for poor outcomes have been reported and used in treatment planning (Creutzberg et al, 2000; Keys et al, 2004; Benedetti Panici et al, 2008; ASTEC study group et al, 2009). Among these is the presence of lymphovascular space invasion (LVSI), which is one of the most important predictors of nodal involvement, recurrence risk, and OS (Morrow et al, 1991; Briët et al, 2005; Guntupalli et al, 2012). The increased demand for clinicians to help patients make informed decisions about treatment options, benefits and risks involves careful insertion of risk factors into the overall treatment recommendations (Creutzberg et al, 2000; Pecorelli, 2009; Colombo et al, 2013). To improve patient risk stratification, the presence of LVSI has been incorporated into international classifications in combination with other clinicopathological factors, such as age, International Federation of Gynecology and Obstetrics (FIGO) stage, pathogenetic types (Bokhman, 1983), histological grade, and depth of myometrial invasion (Keys et al, 2004; Querleu et al, 2011). In contrast, in the European Society of Medical Oncology (ESMO) recurrence risk classification, LVSI is not used to classify patients as being at low, intermediate, or high risk despite its demonstrated prognostic value (Colombo et al, 2013). Furthermore, a considerable heterogeneity of early EC tumour biology and of patients's characteristics within each risk subgroup has been observed (Creutzberg et al, 2000; Keys et al, 2004; Ballester et al, 2011; Nugent et al, 2012).

We hypothesised that in the near future a combination of LVSI status with existing clinico-histopathological features may become an important surrogate risk marker and determinant to better adapt adjuvant therapies in patients with early-stage EC. The aim of this prospective multicentre database study was therefore to investigate whether LVSI may improve the accuracy of the ESMO classification in predicting the recurrence risk in patients with early EC.

\section{MATERIALS AND METHODS}

Study population. Data of all patients with apparent early-stage EC who received primary surgical treatment between January 2001 and December 2012 were abstracted from four institutions with maintained EC databases in France (Tenon University Hospital, Reims University Hospital, Dijon Cancer Center, and Creteil Hospital) and from the Senti-Endo trial (Ballester et al, 2011). All the patients had undergone a preoperative endometrial biopsy. All enrolled patients underwent a preoperative MRI unless contraindicated, in which case a CT scan was performed. Patients with histologically proven EC were staged on the basis of final pathological findings according to the 2009 FIGO classification (Pecorelli, 2009). Clinical and pathological variables included patient age, body mass index (BMI; calculated as weight in kilograms divided by the square of height in meters), surgical procedure, 2009 FIGO stage, and final pathological analysis (histology type and grade, depth of myometrial invasion, and LVSI status). A tumour was considered LVSI-positive when tumour emboli were found within a space clearly lined by endothelial cells (Tsuruchi et al, 1995). The research protocol was approved by the Consultative Committee for Protection of Persons in Biomedical Research of Paris 6 (France).

Treatment and follow-up. Patients were included if they had undergone primary surgical treatment, including at least total hysterectomy with bilateral salpingo-oophorectomy, with or without nodal staging (pelvic $+/-$ para-aortic lymphadenectomy) according to the current guidelines and the surgeon's discretion. If a sentinel lymph node (SLN) biopsy had been undertaken, a dual cervical injection was performed intracervically based on the histological validation of SLN by Delpech et al (2007). If the pelvic SLN was found to be metastatic on intraoperative histology or after final histology, a para-aortic lymphadenectomy was recommended. Systematic pelvic and para-aortic lymphadenectomy was also recommended for patients with type 2 EC (clear-cell, serous EC and carcinosarcoma) and type 1 , grade 3 with myometrial invasion $>50 \%$ EC. Adjuvant therapy was administered on an individual basis at the discretion of the pluridisciplinary committee, based on international guidelines and involved vaginal brachytherapy (VBT) and/or external beam radiotherapy (EBRT) and/or chemotherapy (Colombo et al, 2013). Clinical follow-up consisted of physical examinations and the use of imaging techniques according to the findings. Follow-up sessions were conducted every 3 months for the first 2 years, every 6 months for the following 3 years, and once a year thereafter.

\section{Statistical analysis}

ESMO risk groups and LVSI. Based on definitive histology, three risk groups according to the ESMO guidelines for EC were defined as follows (Colombo et al, 2013): low risk (type $1 \mathrm{EC}$, stage IA grade 1 or 2 ); intermediate risk (type $1 \mathrm{EC}$, stage IA grade 3 , or stage IB grade 1 or 2); and high risk (type $1 \mathrm{EC}$, stage IB grade 3 , or type $2 \mathrm{EC}$ of any stage and grade). A modified ESMO classification including six risk groups (low risk/LVSI - ; low risk/LVSI + ; intermediate risk/LVSI - ; intermediate risk/LVSI +; high risk/ LVSI - ; high risk/LVSI + ) was created after inclusion of the LVSI status in the current ESMO classification.

Recurrence event and recurrence-free survival (RFS). Disease recurrence was diagnosed by biopsy or imaging studies and defined as a relapse without differentiating between their local or distant nature. Survival estimates were based on Kaplan-Meier calculations and RFS defined as the time from the date of surgery to the date of recurrence. Cox proportional hazard models were fitted to independently evaluate the influence of co-variates (age, adjuvant therapy, ESMO classification, and ESMO modified classification). Effects were expressed as hazard ratios with $95 \%$ confidence intervals as appropriate. Log-rank tests were used to examine the individual effects of variables on RFS.

Staging accuracy. The recurrence accuracy of the ESMO and modified ESMO classifications were evaluated with respect to discrimination, that is, whether the relative ranking of individual predictions was in the correct order, quantified with the area under the receiver operating characteristic curve (AUC) (Hanley and McNeil, 1982). The AUC index is the probability that given two randomly selected patients the patient with the worse outcome prediction will have the worse outcome. The AUC index ranges from 0 to 1 , with 1 indicating perfect concordance and 0.5 indicating no association. 
Other statistical analysis. Statistical analysis was based on Student's $t$-test and the Mann-Whitney test for parametric and nonparametric continuous variables, respectively, and the Chisquare test or Fisher's exact test, as appropriate, for categorical variables. Values of $P<0.05$ were considered to denote significant differences. Data were managed with an Excel database (Microsoft, Redmond, WA, USA) and analysed using the R 2.15 software, available online.

\section{RESULTS}

Characteristics of the study population. During the study period, 496 patients with EC were documented as having received primary surgical treatment according to the following distribution: Dijon Cancer Center $(n=129 ; 26 \%)$, Creteil Hospital $(n=97 ; 20 \%)$, Reims University Hospital $(n=92 ; 19 \%)$, Tenon University Hospital $(n=59 ; 12 \%)$, and Senti-Endo trial $(n=119 ; 24 \%)$. The demographics and clinicopathological characteristics of the whole cohort are reported in Table 1 . The median age and BMI of the patients were 67.7 years (range $33-98$ years) and $27.7 \mathrm{~kg} \mathrm{~m}^{-2}$ (range 16.8-48.8), respectively. Postoperative pathogenetic types (Bokhman, 1983) was $83 \%$ (415) and 16\% (77) for type 1 and 2, respectively. Forty-four percent $(n=213), 32 \%(156)$, and $23 \%$ (127) of patients were at low, intermediate, and high risk according to the ESMO classification, respectively. Comparatively, 43\% (213), $18 \%$ (91), 13\% (65), 12\% (58), and 14\% (69) of patients were at low risk, intermediate risk/LVSI - , intermediate risk/LVSI + , high risk/LVSI - , and high risk/LVSI + according to the modified ESMO classification, respectively. Surgical approach consisted of nodal staging for $79.7 \%$ (331 out of 415) and SLNB for 53.7\% (178 out of 331). One hundred and forty-four patients (29\%) were found to have LVSI on final histology. Epidemiological, surgical, histological, and outcome characteristics of patients according to LVSI status are reported in Table 2.

Recurrence and lymph node metastasis rates according to the ESMO and the modified ESMO classifications. The recurrence rate in the whole population was $16.1 \%$ (80 out of 496). When comparing patients with and without LVSI, the recurrence rates were $30.6 \%$ (44 out of 144) vs $10.2 \%$ (36 out of 352 ), respectively $(P<0.001)$. When considering each subgroup of the ESMO classification, the recurrence rates were $6.5 \%$ (14 out of 213), $14.1 \%$ (23 out of 163), and 35.8\% (43 out of 120) for low-, intermediate-, and high-risk patients, respectively $(P<0.001)$. When considering each subgroup of the ESMO modified classification, the recurrence rates were $7.0 \%$ (14 out of 198), $0 \%$ ( 0 out of 15$), 8.2 \%$ ( 8 out of 98 ), $23.1 \%$ (15 out of 65 ), $25.9 \%$ (15 out of 58 ), and $45.1 \%$ (28 out of 62 ) for low risk/LVSI - , low risk/LVSI + , intermediate risk/LVSI - , intermediate risk/LVSI + , high risk/LVSI - , and high risk/LVSI + , respectively $(P<0.001)$. Lymph node metastases were found, respectively, in $6.6 \%$ (12 out of 181), 20\% (25 out of 125), and 33\% (34 out of 102) for low-, intermediate-, and high-risk patients of the ESMO classification $(P<0.001)$. Using the ESMO modified classification, lymph node metastases were found, respectively, in 6.6\% (12 out of 181 ), $10 \%$ (7 out of 70 ), $32.7 \%$ (18 out of 55 ), 17.6\% ( 9 out of 51 ), and $49 \%$ (25 out of 51) for low risk, intermediate risk/LVSI - , intermediate risk/LVSI + , high risk/LVSI - , and high risk/LVSI + $(P<0.001)$.

RFS according to the ESMO and the ESMO modified classifications. The median follow-up and initial recurrence time were 31 (range: 1-152) and 27 (range: 1-134) months, respectively. The respective 5 -year RFS rates according to the ESMO classification were $91.4 \%$ (95\% CI, 86.8-96.2), 77.8\% (95\% CI, 69.7-86.7), and 48.5\% (95\% CI, 37.2-63.3) $(P<0.0001)$ for low-, intermediate-, and high-risk patients (Figure 1). The subset analysis of the ESMO modified
Table 1. Characteristics of the whole population

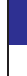
\begin{tabular}{|l|c|}
\hline Characteristics & $\begin{array}{c}\text { Population } \\
\%(\boldsymbol{n}) \\
\boldsymbol{n}=\mathbf{4 9 6}\end{array}$ \\
\hline Age (years), median (range) & $67.7(33-98)$ \\
\hline BMI (kg m ${ }^{-2}$ ), median (range) & $27.7(16.8-48.8)$ \\
\hline
\end{tabular}

Histological type at final histology

\begin{tabular}{|l|r}
\hline Type 1 & $83 \%(415)$ \\
Type 2 & $17 \%(81)$
\end{tabular}

ESMO risk classification

\begin{tabular}{l|l}
\hline Low risk & $44 \%(213)$ \\
Intermediate risk & $32 \%(156)$ \\
High risk & $24 \%(127)$
\end{tabular}

ESMO modified risk classification

\begin{tabular}{|l|c|}
\hline Low risk/LVSI - & $40 \%(198)$ \\
Low risk/LVSI + & $3 \%(15)$ \\
Intermediate risk/LVSI - & $18 \%(91)$ \\
Intermediate risk/LVSI + & $13 \%(65)$ \\
High risk/LVSI - & $12 \%(58)$ \\
High risk/LVSI + & $14 \%(69)$ \\
\hline LVSI & \\
\hline Yes & $29 \%(144)$ \\
No & $71 \%(352)$
\end{tabular}

Nodal staging

Nodal staging (P/PAL)

SLN biopsy

$79.7(331 / 415)$

FIGO stage at definitive histology

\begin{tabular}{|l|c}
\hline IA & $45 \%(225)$ \\
IB & $26 \%(131)$ \\
II & $8 \%(38)$ \\
IIIA & $5 \%(23)$ \\
IIIB & $1 \%(5)$ \\
IIIC & $13 \%(66)$ \\
IV & $2 \%(8)$
\end{tabular}

Adjuvant therapy, n (\%)

Vaginal brachytherapy

EBRT

Chemotherapy

$260 / 496$

$192 / 496$

$80 / 496$

\section{Overall}

Recurrence

Lymph node metastasis

$16.1 \%(80 / 496)$

$17.1 \%(71 / 415)$

Abbreviations: $\mathrm{BMI}=$ body mass index; $\mathrm{EBRT}=$ external beam radiotherapy; $\mathrm{ESMO}=$ European Society of Medical Oncology; FIGO = International Federation of Gynecology and Obstetrics; LVSI = lymphovascular space invasion; $\mathrm{P} / \mathrm{PAL}=$ pelvic/para-aortic lymphadenectomy; $S L N=$ sentinel lymph node.

classification according to the LVSI status reported 5-year RFS of $87.3 \% \quad(95 \%$ CI, 79.3-96.2) vs $63.2 \% \quad$ (95\% CI, $48.5-82.3)$ for intermediate risk/LVSI - and intermediate risk/LVSI + $(P=0.0144)$ and $59.5 \%(95 \% \mathrm{CI}, 42.4-83.6)$ vs $36.9 \%(95 \% \mathrm{CI}$, 23.6-57.8) for high risk/LVSI -, high risk/LVSI + patients, respectively $(P=0.0142$; Figure 2$)$.

Multivariate analysis. In a multivariate analysis including the ESMO classification risk groups, adjuvant therapies (VBT, EBRT, 


\begin{tabular}{|c|c|c|c|}
\hline & $\begin{array}{c}\text { No LVSI } \\
n=352 \\
\%(n)\end{array}$ & $\begin{array}{c}\text { LVSI } \\
n=144 \\
\%(n)\end{array}$ & $P$-value \\
\hline Age (years), mean (range) & $65.9(33-92)$ & $67.4(40-98)$ & 0.17 \\
\hline $\mathrm{BMI}\left(\mathrm{kg} \mathrm{m}^{-2}\right)$, mean (range) & $29.1(16.9-40.2)$ & $28.7(16.9-40.3)$ & 0.65 \\
\hline Follow-up mean (range), months & $35.9(1-134)$ & $30.4(0-152)$ & 0.017 \\
\hline \multicolumn{4}{|l|}{ Histological type } \\
\hline $\begin{array}{l}\text { Type } 1 \\
\text { Type } 2\end{array}$ & $\begin{array}{c}87.7 \%(309) \\
12.3 \%(43)\end{array}$ & $\begin{array}{c}73.6 \%(106) \\
26.4 \%(38)\end{array}$ & 0.0003 \\
\hline \multicolumn{4}{|l|}{ ESMO risk classification } \\
\hline $\begin{array}{l}\text { Low risk } \\
\text { Intermediate risk } \\
\text { High risk }\end{array}$ & $\begin{array}{l}56.2 \%(198) \\
25.9 \%(91) \\
17.9 \%(63)\end{array}$ & $\begin{array}{l}10.4 \%(15) \\
45.1 \%(65) \\
44.4 \%(64)\end{array}$ & $<0.0001$ \\
\hline Nodal staging & $77.8 \%(228 / 293)$ & $84.4 \%(103 / 122)$ & 0.12 \\
\hline Nodal metastasis & $8.5 \%(25 / 293)$ & $38.5 \%(47 / 122)$ & $<0.0001$ \\
\hline Vaginal brachytherapy & $53.1 \%(187)$ & $50.7 \%(73)$ & 0.4366 \\
\hline EBRT & $28.4 \%(100)$ & $63.9 \%(92)$ & $<0.0001$ \\
\hline Chemotherapy & $9.9 \%(35)$ & $31.2(45)$ & $<0.0001$ \\
\hline Recurrence rates & $10.8(36)$ & $30.6(44)$ & $<0.0001$ \\
\hline
\end{tabular}

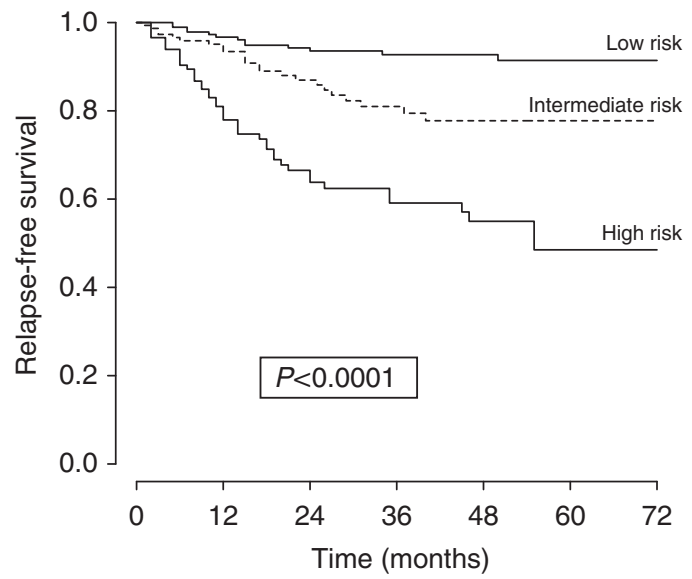

Figure 1. Recurrence-free survival according to ESMO risk classification.

and chemotherapy), and nodal staging, we found that adjuvant chemotherapy $(\mathrm{HR}=2.47(95 \% \mathrm{CI}, 1.48-4.11))$ and nodal staging $(\mathrm{HR}=0.43(95 \% \mathrm{CI}, 0.22-0.84))$ were independently correlated to 5 -year RFS. In a multivariate analysis including the modified ESMO classification risk groups, adjuvant therapies (VBRT, EBRT, and chemotherapy), and nodal staging, we found that intermediate risk/LVSI $+(\mathrm{HR}=2.64(95 \% \mathrm{CI}, 1.18-5.89), P=0.0171)$, high risk/LVSI $-(\mathrm{HR}=2.69(95 \% \mathrm{CI}, 1.18-6.14), P=0.0180)$, high risk/LVSI $+(\mathrm{HR}=4.92(95 \% \mathrm{CI}, 2.34-10.33), P=0.001)$, and adjuvant chemotherapy $(\mathrm{HR}=2.36 \quad(95 \% \quad \mathrm{CI}, 1.42-3.93)$, $P<0.001$ ) were independently correlated to 5-year RFS (Table 3 ).

Staging accuracy. The staging accuracy according to AUC criteria for ESMO and ESMO modified classifications were 0.71 (95\% CI: $0.68-0.74)$ and 0.74 (95\% CI: 0.71-0.77), respectively (Figure 3).

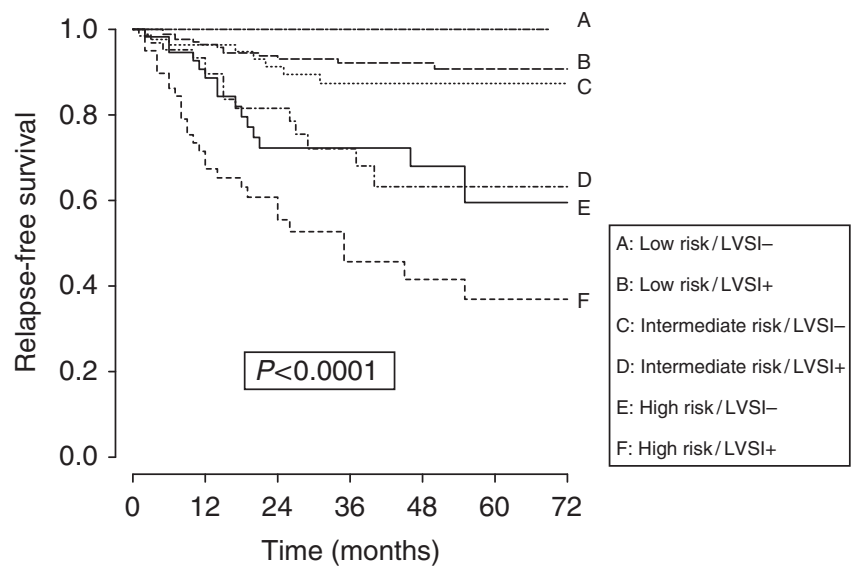

Figure 2. Recurrence-free survival according to modified ESMO risk classification.

\section{DISCUSSION}

Our results demonstrate that the recurrence risk stratification in patients with early-stage EC can be improved by the inclusion of the LVSI status in the current ESMO classification, especially for patients with intermediate risk EC.

In the past decade, LVSI status has progressively gained importance as an independent intrauterine risk factor for patients with early-stage EC (Morrow et al, 1991; Briët et al, 2005; Guntupalli et al, 2012). It is currently considered to be one of the first steps in the metastatic spread even for patients with tumours that seem to be confined to the uterus. In previous studies, the presence of LVSI has been reported as a strong independent prognostic marker with respect to disease recurrence, nodal metastasis, and survival (Morrow et al, 1991; 
Table 3. Multivariate analysis of recurrence-free survival of the modified ESMO classification

RFS

\begin{tabular}{|l|c|c|}
\hline Covariate & HR $(95 \% \mathrm{Cl})$ & $P$ \\
\hline Vaginal brachytherapy \\
\hline $\begin{array}{l}\text { Not performed } \\
\text { Performed }\end{array}$ & $\begin{array}{c}\text { Reference } \\
0.69(0.43-1.10)\end{array}$ & 0.1217 \\
\hline EBRT &
\end{tabular}

\begin{tabular}{|l|c|c|}
\hline $\begin{array}{l}\text { Not performed } \\
\text { Performed }\end{array}$ & $\begin{array}{c}\text { Reference } \\
1.27(0.74-2.17)\end{array}$ & 0.3697 \\
\hline
\end{tabular}

Nodal staging

Not performed

Performed

Reference

$0.44(0.22-0.85)$

0.0159

\section{Chemotherapy}

Not performed
Performed

Reference

2.36 (1.42-3.93)

$<0.001$

\section{Modified ESMO}

\section{Low risk/LVSI -}

Low risk/LVSI +

Intermediate risk/LVSI -

Intermediate risk/LVSI +

High risk/LVSI -

High risk/LVSI +

\begin{tabular}{l|l}
\multicolumn{1}{c|}{ Reference } & \\
$1.05(0.35-1.66)$ & 0.9952 \\
$1.15(0.41-2.68)$ & 0.9110 \\
$2.64(1.18-5.89)$ & 0.0171 \\
$2.69(1.18-6.14)$ & 0.0180 \\
$4.92(2.34-10.33)$ & 0.001
\end{tabular}

Abbreviations: $\mathrm{Cl}=$ confidence interval; $\mathrm{EBRT}=$ external beam radiotherapy; $\mathrm{ESMO}=$ European Society of Medical Oncology; HR=hazard ratio; LVSI=lymphovascular space invasion; RFS = recurrence-free survival.

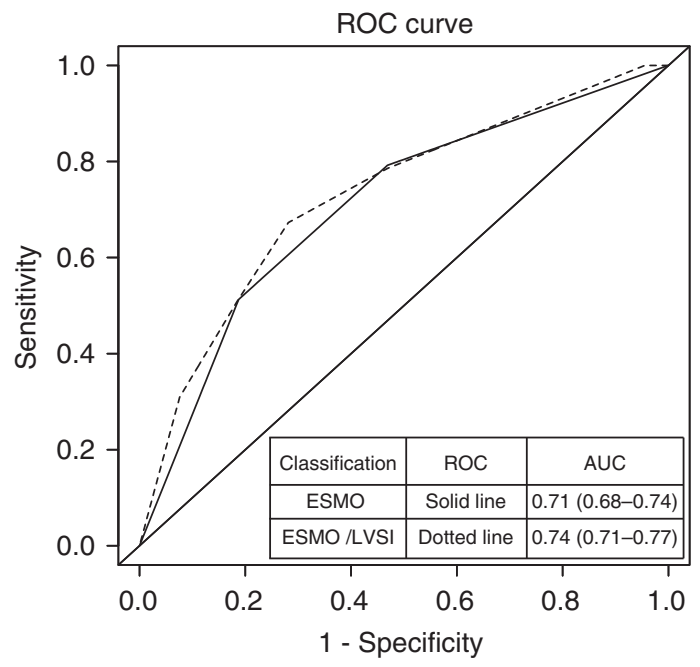

Figure 3. Receiver operating characteristic curves of the ESMO and modified ESMO risk classifications.

Guntupalli et al, 2012). Indeed, Briët et al (2005) reported that LVSI status is related to an increased risk of pelvic lymph node metastasis and higher rates of recurrence. In this specific setting, Laufer et al (2013) showed that LVSI emerged as the strongest independent variable associated with pelvic lymph node metastasis compared with histological grade and depth of myometrial invasion. Similarly, both Guntupally et al (2012) and Simpkins et al (2013) demonstrate that LVSI is highly associated with LN metastasis and is a strong marker of local and distant disease recurrence. Our results are thus in complete accordance with these previous results, showing that LVSI status is an independent prognostic marker for recurrence and decreased RFS.

There are several unresolved issues with regards to accurately classifying the risk of recurrence of early-stage EC and the impact on indications for adjuvant therapies. Previous efforts of risk grouping have led to the development of recommendations in which several reliable factors were combined (Creutzberg et al, 2000; Keys et al, 2004; Colombo et al, 2013). The main interest of such an approach is to stratify patients not only to plan surgical or adjuvant treatment but also to provide better information to patients about treatment options. Although these approaches constitute a more reliable estimation of the risk of recurrence than the physician's judgment, their predictive capability is based on the assumption that all patients within a given risk group are equal. However, in clinical practice, both biological heterogeneity of endometrial tumours and heterogeneity of patients's characteristics within each subgroup have been observed, especially in early-stage EC (Creutzberg et al, 2000; Keys et al, 2004; Ballester et al, 2011, 2012). The current ESMO classification established three risk groups of recurrence based on depth of myometrial invasion, histological grade, and type, without taking into account the LVSI status (Colombo et al, 2013). In the present study, we hypothesised that LVSI status may improve the ESMO accuracy. Indeed, the introduction of LVSI status within the current ESMO classification resulted in an improvement of its discrimination accuracy with an AUC of 0.74 . Moreover, we found that patients with IR/LVSI + EC had similar recurrence rates and RFS to those with HR/LVSI - . This is in accordance with the GOG-99 study that established a high-intermediate risk (HIR) group for recurrence based on the LVSI status, invasion of tumour into the outer one-third of the myometrium, and histological grade $2-3$. The authors found that, in the intermediate HIR risk group, $21 \%$ of patients with negative LN and $42 \%$ of patients with positive LN experienced a recurrence (Keys et al, 2004).

There is a persistent debate about the relevance of lymphadenectomy in standard management of EC despite the publication of two randomised trials showing that there is no advantage in performing systematic lymphadenectomy in this setting (Benedetti Panici et al, 2008; ASTEC study group et al, 2009). Currently, the ESMO group recommends a hysterectomy with bilateral salpingooophorectomy \pm bilateral pelvic-para-aortic lymphadenectomy, underlining that indications for nodal staging in this specific subgroup are somewhat blurred (Colombo et al, 2013). In our study, we found that $32.7 \%$ of patients with IR/LVSI + had nodal metastases ( $v s 17.6 \%$ in the HR/LVSI - ), with a recurrence rate of $27.7 \%$. In contrast, we found that $10 \%$ of patients with IR/LVSI had nodal metastases, with a recurrence rate of $14.2 \%$. These results compare favourably with those of Nugent et al (2012), strongly suggesting that information on nodal status cannot be omitted in this subgroup of patients to better adapt adjuvant therapies. This is in accordance with the results of the Senti-Endo study showing that $15 \%$ of patients with intermediate-risk EC have LN metastases that could go undetected if there is no nodal staging (Ballester et al, 2011). Hence, SLN biopsy appears as a relevant alternative for the IR/LVSI + risk group of recurrence. Moreover, because of the high rate of discrepancies between preoperative and postoperative risk groups, comprehensive lymphadenectomy can always be performed later.

Another topic of controversy is related to indications for adjuvant therapies in patients with early-stage EC. For intermediate-risk EC, the current ESMO guidelines recommend observation or VBT (Colombo et al, 2013). In the case of negative prognostic factors, pelvic EBRT and/or adjunctive chemotherapy could be considered. In HIR patients with early-stage EC, 
the PORTEC-1 trial found that EBRT decreased local recurrence rates from $14 \%$ to $4 \%(P=0.001)$, with no difference in OS $(81 \%$ 5 -year survival in the radiotherapy group vs $85 \%, P=0.31$ ) or in the development of distant metastatic disease (Creutzberg et al, 2000). These results were confirmed by the GOGG 99 trial in patients who had undergone systematic lymphadenectomy (Keys et al, 2004). These findings, coupled with our results, suggest that among patients with intermediate risk a subset of patients with negative prognostic factors, such as our subgroup of patients with IR/LVSI + without LN metastases, could benefit from EBRT.

Intraoperative assessment of the LVSI status may allow a more precise stratification of these tumours and therefore remains a very important clinical challenge. However, its role in decision making for surgical staging of EC remains controversial (Guntupalli et al, 2012; Laufer et al, 2013; Simpkins et al, 2013). Indeed, as previously reported, the intraoperative assessment is based on a limited sample and may not be in agreement with the final pathology (Guntupalli et al, 2012; Laufer et al, 2013; Simpkins et al, 2013). Hence, further studies are needed to confirm its clinical value.

The strengths of our study reside in its multicentre design and the large number of patients as we were able to demonstrate the prognostic weight of the LVSI and the accuracy of the ESMO modified by the incorporation of LVSI. However, some weaknesses deserve to be mentioned. First, there is the inherent selection bias found at large multicentre tertiary referral centres. Second, during the data collection period, modifications occurred in staging modalities (FIGO classification; Pecorelli, 2009) and surgical techniques (LN staging; Benedetti Panici et al, 2008; ASTEC study group et al, 2009; Ballester et al, 2011). Third, our analysis does not take into account some histological characteristics, such as the tumour size. Because of the low number of event recurrence, we were unable to perform an analysis according to locoregional and distant recurrence. Fourth, to avoid any interpretation bias, we did not assess the significance of isolated tumour cells and the prognostic value of the number of LVSI. Finally, we could not demonstrate a statistical difference in low-risk patients according to the LVSI status, probably due to the low incidence of LVSI in this subgroup of patients (type 1/low grade tumours). However, O'Brien et al (2009) reported that in patients with early-stage welldifferentiated adenocarcinoma of the endometrium, the presence of LVSI is associated with a high risk of death. In addition, the latest recommendations on EC elaborated in France have used a modified classification that takes into account the presence of LVSI to define LVSI + as high-risk EC (Querleu et al, 2011).

In conclusion, our results confirm that LVSI appears to be highly predictive of disease recurrence and decreased RFS in early stage EC. Furthermore, the inclusion of the LVSI status into the current ESMO classification improves its accuracy, especially in patients with intermediate-risk early-stage EC. This criterion should be taken into consideration to better adapt indications for nodal staging and adjuvant therapies. In the future, improving health-care management could be achieved by combining the molecular (Dai et al, 2014) and immunohistochemical biomarkers (Ballester et al, 2013) with current risk factors to provide more precise information that will allow for more individualised treatments.

\section{CONFLICT OF INTEREST}

The authors declare no conflict of interest.

\section{REFERENCES}

ASTEC study groupKitchener H, Swart AMC, Qian Q, Amos C, Parmar MKB (2009) Efficacy of systematic pelvic lymphadenectomy in endometrial cancer (MRC ASTEC trial): a randomised study. Lancet 373: 125-136.
Ballester M, Canlorbe G, Cortez A, Gonin J, Laas E, Bendifallah S, Graesslin O, Daraï E (2013) Histological and immunohistochemical profiles predict lymph node status in women with low-intermediate risk endometrial cancer. Gynecol Oncol 130: 457-462.

Ballester M, Dubernard G, Bats A-S, Heitz D, Mathevet P, Marret H, Querleu D, Golfier F, Leblanc E, Rouzier R, Daraï E (2012) Comparison of diagnostic accuracy of frozen section with imprint cytology for intraoperative examination of sentinel lymph node in early-stage endometrial cancer: results of Senti-Endo study. Ann Surg Oncol 19: 3515-3521.

Ballester M, Dubernard G, Lécuru F, Heitz D, Mathevet P, Marret H, Querleu D, Golfier F, Leblanc E, Rouzier R, Daraï E (2011) Detection rate and diagnostic accuracy of sentinel-node biopsy in early stage endometrial cancer: a prospective multicentre study (SENTI-ENDO). Lancet Oncol 12: $469-476$.

Benedetti Panici P, Basile S, Maneschi F, Alberto Lissoni A, Signorelli M, Scambia G, Angioli R, Tateo S, Mangili G, Katsaros D, Garozzo G, Campagnutta E, Donadello N, Greggi S, Melpignano M, Raspagliesi F, Ragni N, Cormio G, Grassi R, Franchi M, Giannarelli D, Fossati R, Torri V, Amoroso M, Crocè C, Mangioni C (2008) Systematic pelvic lymphadenectomy vs. no lymphadenectomy in early-stage endometrial carcinoma: randomized clinical trial. J Natl Cancer Inst 100: 1707-1716.

Bokhman JV (1983) Two pathogenetic types of endometrial carcinoma. Gynecol Oncol 15: 10-17.

Briët JM, Hollema H, Reesink N, Aalders JG, Mourits MJE, ten Hoor KA, Pras E, Boezen HM, van der Zee AGJ, Nijman HW (2005) Lymphvascular space involvement: an independent prognostic factor in endometrial cancer. Gynecol Oncol 96: 799-804.

Colombo N, Preti E, Landoni F, Carinelli S, Colombo A, Marini C, Sessa C. ESMO Guidelines Working Group (2013) Endometrial cancer: ESMO Clinical Practice Guidelines for Diagnosis, Treatment and Follow-Up. Ann Oncol Off J Eur Soc Med Oncol ESMO 24(Suppl 6): vi33-vi38.

Creasman WT, Morrow CP, Bundy BN, Homesley HD, Graham JE, Heller PB (1987) Surgical pathologic spread patterns of endometrial cancer. A Gynecologic Oncology Group Study. Cancer 60: 2035-2041.

Creasman WT, Odicino F, Maisonneuve P, Quinn MA, Beller U, Benedet JL, Heintz APM, Ngan HYS, Pecorelli S (2006) Carcinoma of the corpus uteri. FIGO 26th Annual Report on the Results of Treatment in Gynecological Cancer. Int J Gynaecol Obstet Off Organ Int Fed Gynaecol Obstet 95(Suppl 1): S105-S143.

Creutzberg CL, van Putten WL, Koper PC, Lybeert ML, Jobsen JJ, Wárlám-Rodenhuis CC, De Winter KA, Lutgens LC, van den Bergh AC, van de Steen-Banasik E, Beerman H, van Lent M (2000) Surgery and postoperative radiotherapy versus surgery alone for patients with stage-1 endometrial carcinoma: multicentre randomised trial. PORTEC Study Group. Post Operative Radiation Therapy in Endometrial Carcinoma. Lancet 355: 1404-1411.

Dai D, Bradford AP, Prossnitz ER (2014) Endometrial cancer: molecular and cellular basis of tumor development, novel biomarkers and therapeutic agents, and innovative research approaches. Obstet Gynecol Int 2014: 710405.

Delpech Y, Cortez A, Coutant C, Callard P, Uzan S, Darai E, Barranger E (2007) The sentinel node concept in endometrial cancer: histopathologic validation by serial section and immunohistochemistry. Ann Oncol Off J Eur Soc Med Oncol ESMO 18: 1799-1803.

Guntupalli SR, Zighelboim I, Kizer NT, Zhang Q, Powell MA, Thaker PH, Goodfellow PJ, Mutch DG (2012) Lymphovascular space invasion is an independent risk factor for nodal disease and poor outcomes in endometrioid endometrial cancer. Gynecol Oncol 124: 31-35.

Hanley JA, McNeil BJ (1982) The meaning and use of the area under a receiver operating characteristic (ROC) curve. Radiology 143: 29-36.

Jemal A, Siegel R, Xu J, Ward E (2010) Cancer statistics, 2010. CA Cancer $J$ Clin 60: 277-300.

Keys HM, Roberts JA, Brunetto VL, Zaino RJ, Spirtos NM, Bloss JD, Pearlman A, Maiman MA, Bell JG. Gynecologic Oncology Group (2004) A phase III trial of surgery with or without adjunctive external pelvic radiation therapy in intermediate risk endometrial adenocarcinoma: a Gynecologic Oncology Group study. Gynecol Oncol 92: 744-751.

Laufer J, Scasso S, Papadia A, Sosa C, Cirillo F, Raspagliesi F (2013) Association between tumor diameter and lymphovascular space invasion among women with early-stage endometrial cancer. Int J Gynaecol Obstet Off Organ Int Fed Gynaecol Obstet 123: 142-145.

Mariani A, Webb MJ, Keeney GL, Lesnick TG, Podratz KC (2002) Surgical stage I endometrial cancer: predictors of distant failure and death. Gynecol Oncol 87: 274-280. 
Morrow CP, Bundy BN, Kurman RJ, Creasman WT, Heller P, Homesley HD, Graham JE (1991) Relationship between surgical-pathological risk factors and outcome in clinical stage I and II carcinoma of the endometrium: a Gynecologic Oncology Group study. Gynecol Oncol 40: 55-65.

Nout RA, Smit VTHBM, Putter H, Jürgenliemk-Schulz IM, Jobsen JJ, Lutgens LCHW, van der Steen-Banasik EM, Mens JWM, Slot A, Kroese MCS, van Bunningen BNFM, Ansink AC, van Putten WLJ, Creutzberg CL. PORTEC Study Group (2010) Vaginal brachytherapy versus pelvic external beam radiotherapy for patients with endometrial cancer of high-intermediate risk (PORTEC-2): an open-label, noninferiority, randomised trial. Lancet 375: 816-823.

Nugent EK, Bishop EA, Mathews CA, Moxley KM, Tenney M, Mannel RS, Walker JL, Moore KN, Landrum LM, McMeekin DS (2012) Do uterine risk factors or lymph node metastasis more significantly affect recurrence in patients with endometrioid adenocarcinoma? Gynecol Oncol 125: 94-98.

O’Brien DJ, Flannelly G, Mooney EE, Foley M (2009) Lymphovascular space involvement in early stage well-differentiated endometrial cancer is associated with increased mortality. BJOG Int J Obstet Gynaecol 116: 991-994.

Pecorelli S (2009) Revised FIGO staging for carcinoma of the vulva, cervix, and endometrium. Int J Gynaecol Obstet Off Organ Int Fed Gynaecol Obstet 105: 103-104.

Querleu D, Planchamp F, Narducci F, Morice P, Joly F, Genestie C, Haie-Meder C, Thomas L, Quénel-Tueux N, Daraï E, Dorangeon P-H, Marret H, Taïeb S, Mazeau-Woynar V. Institut National du Cancer, Societe Francaise d'Oncologie Gynecologique (2011) Clinical practice guidelines for the management of patients with endometrial cancer in France: recommendations of the Institut National du Cancer and the Société Française d'Oncologie Gynécologique. Int J Gynecol Cancer Off J Int Gynecol Cancer Soc 21: 945-950.

Randall ME, Filiaci VL, Muss H, Spirtos NM, Mannel RS, Fowler J, Thigpen JT, Benda JA. Gynecologic Oncology Group Study (2006) Randomized phase III trial of whole-abdominal irradiation versus doxorubicin and cisplatin chemotherapy in advanced endometrial carcinoma: a Gynecologic Oncology Group Study. J Clin Oncol Off J Am Soc Clin Oncol 24: 36-44.

Simpkins F, Papadia A, Kunos C, Michener C, Frasure H, AbuShahin F, Mariani A, Bakkum-Gamez JN, Landrum L, Moore K, Thomas SG, Windhorn A, Rose PG (2013) Patterns of recurrence in stage I endometrioid endometrial adenocarcinoma with lymphovascular space invasion. Int J Gynecol Cancer Off J Int Gynecol Cancer Soc 23: 98-104.

Tsuruchi N, Kaku T, Kamura T, Tsukamoto N, Tsuneyoshi M, Akazawa K, Nakano H (1995) The prognostic significance of lymphovascular space invasion in endometrial cancer when conventional hemotoxylin and eosin staining is compared to immunohistochemical staining. Gynecol Oncol 57: $307-312$.

This work is published under the standard license to publish agreement. After 12 months the work will become freely available and the license terms will switch to a Creative Commons AttributionNonCommercial-Share Alike 3.0 Unported License. 